\title{
Phototrophic Fe(II) oxidation takes place in organic rich environments
}

\author{
VERENA NIKELEIT ${ }^{1}$, MARKUS MAISCH ${ }^{1}$, JAMES M. \\ BYRNE $^{2}$, CAROLINE HARWOOD ${ }^{3}$, ANDREAS KAPPLER ${ }^{1}$ \\ AND CASEY BRYCE ${ }^{2}$ \\ ${ }^{1}$ University of Tuebingen \\ ${ }^{2}$ University of Bristol \\ ${ }^{3}$ University of Washington \\ Presenting Author: verena.nikeleit@uni-tuebingen.de
}

Rhodopseudomonas palustris TIE-1, a phototrophic nonsulphur bacterium can utilize $\mathrm{Fe}$ (II) as an electron donor to generate reducing equivalents to fix $\mathrm{CO}_{2}$ and build biomass. Additionally $R$. palustris TIE-1 can also grow photoheterotrophically with a variety of organic substrates like acetate, lactate, pyruvate, butyrate and glucose. However, it is unclear whether such organisms would conduct Fe(II) oxidation in environments with abundant organic substrates. To investigate the contribution of anoxygenic phototrophs to the iron cycle in organic-rich environments, we tested the substrate preference of $R$. palustris TIE-1. In batch experiments $R$. palustris TIE-1 was incubated with $\mathrm{FeCl}_{2}$ and an additional organic substrate. Experiments were conducted both in the presence and absence of an external $\mathrm{CO}_{2}$ source i.e with either $\mathrm{HCO}_{3}{ }^{-}$or PIPES buffer. With PIPES buffer only the organic substrates acetate, lactate and pyruvate were consumed. When $\mathrm{CO}_{2}$ was present, iron and all organic substrates were consumed but the timing and rates of consumption were substrate-dependent. With acetate, butyrate and pyruvate sequential consumption and with lactate and glucose simultaneous consumption with iron were observed. The presence of $\mathrm{Fe}$ also accelerated the consumption rate of the organics, especially lactate. The presence of an organic substrate had no impact on iron mineralogy. This study demonstrates that $\mathrm{Fe}(\mathrm{II})$ oxidation proceeds in the presence of organics, and that $\mathrm{Fe}(\mathrm{II})$ is even beneficial in a number of scenarios. 